\title{
RELATIONSHIP OF PARENTAL HEALTH AND SOCIAL SUPPORT DURING PREGNANCY WITH THE RISK OF NON-SYNDROMIC ORAL CLEFTS: A CASE - CONTROL STUDY
}

\author{
Saira Afzal, ${ }^{1}$ Mustehsan Bashir, ${ }^{2}$ Muhammad Arif Khan, ${ }^{3}$ Javaria Tehzeeb, ${ }^{4}$ Anum Manzoor ${ }^{5}$ \\ Aaila Sajid Dar, ${ }^{6}$ Muhammad Umair, ${ }^{7}$ Abeera Ishaq Butt, ${ }^{8}$ Aroobah Iqbal, ${ }^{9}$ Aniqa Anwar ${ }^{10}$ \\ M. Jawad Bashir, ${ }^{11}$ M. Mishkat Junaid, ${ }^{12}$ M. Salman Ashraf ${ }^{13}$
}

\begin{abstract}
Oral clefts are divided into cleft lip, cleft palate and cleft lip along with cleft palate. Cleft palate is defined as an inability of palatal shelves to approximate and close during the first months of embryogenic period
\end{abstract}

thus leaving an opening in the roof of the mouth and forming communication between the nasal and oral cavities.

Objective: To determine the relationship of risk of oral clefts with parental health and social support.
Afzal S. ${ }^{1}$

Chairperson and Head of Community Medicine Department

King Edward Medical University Lahore

Bashir M. ${ }^{2}$

Chairman and Head of Plastic Surgery Department

KEMU / Mayo Hospital, Lahore

Khan M.A. ${ }^{3}$

APMO Community Medicine Department

King Edward Medical University, Lahore

Tehzeeb J. ${ }^{4}$

MBBS Student, King Edward Medical University, Lahore

Manzoor A. ${ }^{5}$

MBBS Student, King Edward Medical University, Lahore

Dar A.S. ${ }^{6}$

MBBS Student, King Edward Medical University, Lahore
Umair M. ${ }^{7}$

MBBS Student, King Edward Medical University, Lahore

Butt A.I. $^{8}$

MBBS Student, King Edward Medical University, Lahore

Iqbal A. ${ }^{9}$

MBBS Student, King Edward Medical University, Lahore

Anwar A. ${ }^{10}$

MBBS Student, King Edward Medical University, Lahore

Bashir M.J. ${ }^{11}$

MBBS Student, King Edward Medical University, Lahore

Junaid M.M. $^{12}$

MBBS Student, King Edward Medical University, Lahore

Ashraf M.S. ${ }^{13}$

MBBS Student, King Edward Medical University, Lahore 
Study Design: Case Control Study.

Study Setting and Duration: Various hospitals in Lahore and 3 month duration.

Materials and Methods: A Case Control Study was conducted. A total of 100 subjects (50 cases, 50 controls) were included. Sampling was done by purposive method. Data was collected with the help of Cornell Medical Index Health Questionnaire and Social Support Questionnaire after taking their informed consent. Data was analyzed by using SPSS version 20.0. Mean and standard deviation were used to describe quantitative variables like age. Test of significance were applied.

Results: $56 \%$ of cases were females and $44 \%$ were males. Mean age of mothers was 27 and fathers was 31. Most mothers were uneducated $(36 \%)$ while most fathers were educated till matric $(34 \%)$. Income of most parents was in the range of 10,000-20,000 PKR (44\%). $24 \%$ of controls were females and $76 \%$ were males. The associations of oral cleft was found significant with parental respiratory, cardiovascular, gastrointestinal, musculoskeletal, skin-related, genitourinary health, easy fatigability, health habits, anxiety, anger, paternal nervous system health, sensitivity, and maternal frequency of illness, depression and feeling of inadequacy $(p<0.05)$. Maternal social support categories indicated that cases were less supported than controls: number of persons available and level of satisfaction $(\mathrm{p}<0.05)$.

Conclusion: Oral clefts have a multi-factorial etiology. Hence, an effective preventive program should take into account parental health and social support factors.

Key Words: Non-syndromic Oral Clefts, Parental Health, Social Support.

\section{Introduction}

This is a case - control study to establish the relationship of mental and physical health of both parents and social support both formally and informally by the family and community during pregnancy with nonsyndromic oral clefts.

Oral clefts are divided into cleft lip, cleft palate and cleft lip along with cleft palate. Cleft palate is defined as an inability of palatal shelves to approximate and close during the first months of embryogenic period thus leaving an opening in the roof of the mouth and forming communication between the nasal and oral cavities. ${ }^{1}$ Cleft lip results due to failure of fus- ion of both sides of fetal lip tissue by 35 days of gestation. The defect may affect one side or both. ${ }^{2}$ Esthetic defects which are associated with cleft lip and cleft palate in infants results in feeding problems, infections of ear, hearing impairement, dentition problems, difficulty in articulation, social, psychological and behavioral problems. ${ }^{3}$

The incidence of cleft lip in Pakistan is one in 500 live births. This is fourth largest in the world after China, India and Indonesia. Every day 25 cases in Pakistan are born with this deformity and in a year 9000 cases are added. ${ }^{4}$ Many causative factors are found to be linked with cleft lip and palate. Researchers have majorly outlined risk factors like parental age,${ }^{5-7}$ family history, ${ }^{8}$ consanguineous marriages, ${ }^{9,10}$ co-morbidities like infections during pregnancy, ${ }^{11}$ multigravida $^{12}$ in mothers of low socio-economic status, smoking ${ }^{13}$ alcohol, ${ }^{14}$ drugs $^{15}$ (anti-epileptics, steroids, antineoplastics), occupational and environmental exposures. ${ }^{16}$

A research conducted in China studied oral clefts in relation to parental health and social support during pregnancy. ${ }^{17}$ However, no such study has been conducted in Pakistan.

Our study will outline the parental health and social support factors associated with the said defect. This can help shift the paradigm of approach for prevention of this health problem towards a more holistically oriented one. Moreover, various fruitful research efforts can be based on the results of this research to ascertain the risk factors in case of health of both parents and support of family and society.

\section{Materials and Methods}

The study was conducted in various hospitals of Lahore. Informed consent was taken. The risk and benefits were explained and the personal information of study subjects was kept confidential. At $95 \%$ confidence level the sample size was calculated to be 100 . The sample consisted of 50 cases and 50 controls. It was 1:1. Sampling was done by Purposive Method (non-probability). Oral Clefts: Oral cleft is a fissure in upper lip causing cleft lip and an opening in roof of mouth forming cleft palate. ${ }^{18}$ Syndromic Oral Clefts: Oral clefts which are part of known congenital syndromes. Non-syndromic Oral Clefts: Oral clefts associated with no or one major anomaly or two or fewer minor anomalies. ${ }^{19}$ Case: A patient of non-syndromic oral clefts having age of less than 5 years. Control: 
Any child without any craniofacial anomaly having age of less than 5 years. Cornell Medical Index (CMI): It is a standardized questionnaire which is appropriate for collection of relevant medical and psychiatric information on a large mass with a less consumption of time of a doctor. ${ }^{20}$ Social Support Rating Scale: A standardized questionnaire for collecting pertinent social support data. ${ }^{21}$

The children less or equal to 5years of age were divided in two groups. Patients having Cleft Lip, Cleft Palate or Cleft Lip and Palate is termed as a Case group while patients not having Cleft Lip, Cleft Palate or Cleft Lip and Palate constitute Control group. Patients having single minor associated anomalies except other craniofacial malformations (Patent Ductus Arteriosus, Inguinal hernia, Umbilical hernia, Undescended testes, Hydrocele). ${ }^{22}$ The cases where consent was not granted by the parents are excluded from the study. Pretested and validated tools i.e. Cornell Medical Index Health Questionnaire and Social Support Performa were used. All data was collected, entered and analy- zed with the help of SPSS version 20.0. Value of $p$ less than 0.05 was considered as significant.

\section{Results}

$56 \%$ of cases were females and $44 \%$ were males. Mean age of mothers was 27 and fathers was 31. Most mothers were uneducated $(36 \%)$ while most fathers were educated till matric $(34 \%)$. Income of most parents was in the range of 10,000-20,000 PKR (44\%). $24 \%$ of controls were females and $76 \%$ were males.

The associations of oral cleft was found significant with parental respiratory, cardiovascular, gastrointestinal, musculoskeletal, skin-related, genitourinary health, easy fatigability, health habits, anxiety, anger, paternal nervous system health, sensitivity, and maternal frequency of illness, depression and feeling of inadequacy ( $\mathrm{p}<0.05$ ). Maternal social support categories indicated that cases were less supported than controls: number of persons available and level of satisfaction $(\mathrm{p}<0.05)$.

Table 1: Statistical Analysis of Cornell Medical Indices and Social Support Scores for Males.

\begin{tabular}{|c|c|c|c|c|c|c|c|c|c|}
\hline \multirow{3}{*}{$\begin{array}{l}\text { Pair } \\
\text { No. }\end{array}$} & \multirow{3}{*}{ System Studied } & \multicolumn{5}{|c|}{ Paired Differences } & \multirow{3}{*}{$\mathrm{t}$} & \multirow{3}{*}{$\mathrm{df}$} & \multirow{3}{*}{$\begin{array}{l}\text { Sig. }(2- \\
\text { tailed) }\end{array}$} \\
\hline & & \multirow[t]{2}{*}{ Mean } & \multirow{2}{*}{$\begin{array}{l}\text { Std. } \\
\text { Deviation }\end{array}$} & \multirow{2}{*}{$\begin{array}{l}\text { Std. } \\
\text { Error } \\
\text { Mean }\end{array}$} & \multicolumn{2}{|c|}{$\begin{array}{l}95 \% \text { Confidence } \\
\text { Interval of the } \\
\text { Difference }\end{array}$} & & & \\
\hline & & & & & Lower & Upper & & & \\
\hline 1 & $\begin{array}{l}\text { Eyes and Ears (cases)- } \\
\text { Eyes and Ears (controls) }\end{array}$ & $\begin{array}{l}.70 \\
.60\end{array}$ & $\begin{array}{l}.839 \\
.904\end{array}$ & $\begin{array}{l}.119 \\
.128\end{array}$ & -.255 & .455 & .566 & 49 & .574 \\
\hline 2 & $\begin{array}{l}\text { Respiratory( cases)- } \\
\text { Respiratory (controls) }\end{array}$ & $\begin{array}{r}1.40 \\
.64\end{array}$ & $\begin{array}{l}1.429 \\
1.102\end{array}$ & $\begin{array}{l}.202 \\
.156\end{array}$ & .223 & 1.297 & 2.843 & 49 & .007 \\
\hline 3 & $\begin{array}{l}\text { Cardiovascular (cases)- } \\
\text { Cardiovascular (controls) }\end{array}$ & $\begin{array}{r}1.54 \\
.60\end{array}$ & $\begin{array}{r}1.403 \\
.833\end{array}$ & $\begin{array}{l}.198 \\
.118\end{array}$ & .520 & 1.360 & 4.502 & 49 & .000 \\
\hline 4 & $\begin{array}{l}\text { Gastrointestinal (cases)- } \\
\text { Gastrointestinal (controls) }\end{array}$ & $\begin{array}{r}1.56 \\
.78\end{array}$ & $\begin{array}{l}1.541 \\
1.093\end{array}$ & $\begin{array}{l}.218 \\
.155\end{array}$ & .272 & 1.288 & 3.085 & 49 & .003 \\
\hline 5 & $\begin{array}{l}\text { Musculoskeletal (cases)- } \\
\text { Musculoskeletal (controls) }\end{array}$ & $\begin{array}{l}.82 \\
.32\end{array}$ & $\begin{array}{r}1.044 \\
.741\end{array}$ & $\begin{array}{l}.148 \\
.105\end{array}$ & .110 & .890 & 2.573 & 49 & .013 \\
\hline 6 & Skin (cases)-Skin (controls) & $\begin{array}{l}.66 \\
.20\end{array}$ & $\begin{array}{l}.872 \\
.606\end{array}$ & $\begin{array}{l}.123 \\
.086\end{array}$ & .160 & .760 & 3.086 & 49 & .003 \\
\hline 7 & $\begin{array}{l}\text { Nervous (cases)- } \\
\text { Nervous (controls) }\end{array}$ & $\begin{array}{r}1.04 \\
.48\end{array}$ & $\begin{array}{r}1.355 \\
.614\end{array}$ & $\begin{array}{l}.192 \\
.087\end{array}$ & .153 & .967 & 2.767 & 49 & .008 \\
\hline 8 & $\begin{array}{l}\text { Genitourinary (cases)- } \\
\text { Genitourinary (controls) }\end{array}$ & $\begin{array}{r}1.02 \\
.38\end{array}$ & $\begin{array}{r}1.220 \\
.780\end{array}$ & $\begin{array}{l}.173 \\
.110\end{array}$ & .239 & 1.041 & 3.208 & 49 & .002 \\
\hline
\end{tabular}




\begin{tabular}{|c|c|c|c|c|c|c|c|c|c|}
\hline 9 & $\begin{array}{l}\text { Fatigability (cases)- } \\
\text { Fatigability (controls) }\end{array}$ & $\begin{array}{r}1.08 \\
.36\end{array}$ & $\begin{array}{r}1.291 \\
.776 \\
\end{array}$ & $\begin{array}{l}.183 \\
.110\end{array}$ & .250 & 1.190 & 3.078 & 49 & .003 \\
\hline 10 & $\begin{array}{l}\text { Frequency of illness (cases)- } \\
\text { Frequency of illness } \\
\text { (controls) }\end{array}$ & $\begin{array}{l}.52 \\
.30\end{array}$ & $\begin{array}{l}.863 \\
.839\end{array}$ & $\begin{array}{l}.122 \\
.119\end{array}$ & -.096 & .536 & 1.399 & 49 & .168 \\
\hline 11 & $\begin{array}{l}\text { Miscellaneous diseases } \\
\text { (cases)-Miscellaneous } \\
\text { disease (controls) }\end{array}$ & $\begin{array}{r}1.04 \\
.58\end{array}$ & $\begin{array}{r}1.177 \\
.835\end{array}$ & $\begin{array}{l}.167 \\
.118\end{array}$ & .105 & .815 & 2.605 & 49 & .012 \\
\hline 12 & $\begin{array}{l}\text { Health habits(cases)-Health } \\
\text { habits (controls) }\end{array}$ & $\begin{array}{r}1.08 \\
.58\end{array}$ & $\begin{array}{r}1.322 \\
.859\end{array}$ & $\begin{array}{l}.187 \\
.122\end{array}$ & .054 & .946 & 2.255 & 49 & .029 \\
\hline 13 & $\begin{array}{l}\text { Inadequacy (cases)- } \\
\text { Inadequacy (controls) }\end{array}$ & $\begin{array}{l}.68 \\
.64\end{array}$ & $\begin{array}{l}1.058 \\
1.083\end{array}$ & $\begin{array}{l}.150 \\
.153\end{array}$ & -.341 & .421 & .211 & 49 & .834 \\
\hline 14 & $\begin{array}{l}\text { Depression(cases)- } \\
\text { Depression (controls) }\end{array}$ & $\begin{array}{l}.46 \\
.32\end{array}$ & $\begin{array}{r}.734 \\
1.096\end{array}$ & $\begin{array}{l}.104 \\
.155\end{array}$ & -.214 & .494 & .795 & 49 & .431 \\
\hline 15 & $\begin{array}{l}\text { Anxiety (cases)- } \\
\text { Anxiety (controls) }\end{array}$ & $\begin{array}{l}.74 \\
.24\end{array}$ & $\begin{array}{r}1.175 \\
.687 \\
\end{array}$ & $\begin{array}{l}.166 \\
.097 \\
\end{array}$ & .110 & .890 & 2.573 & 49 & .013 \\
\hline 16 & $\begin{array}{l}\text { Sensitivity (cases)- } \\
\text { Sensitivity (controls) }\end{array}$ & $\begin{array}{l}.86 \\
.30\end{array}$ & $\begin{array}{r}1.050 \\
.839\end{array}$ & $\begin{array}{l}.148 \\
.119\end{array}$ & 192 & .928 & 3.055 & 49 & .004 \\
\hline 17 & $\begin{array}{l}\text { Anger (cases)- } \\
\text { Anger (controls) }\end{array}$ & $\begin{array}{l}1.94 \\
1.18\end{array}$ & $\begin{array}{l}1.845 \\
1.781\end{array}$ & $\begin{array}{l}.261 \\
.252\end{array}$ & .137 & 1.383 & 2.453 & 49 & .018 \\
\hline 18 & $\begin{array}{l}\text { Tension (cases)- } \\
\text { Tension (controls) }\end{array}$ & $\begin{array}{l}.70 \\
.22\end{array}$ & $\begin{array}{r}1.055 \\
.616\end{array}$ & $\begin{array}{l}.149 \\
.087\end{array}$ & .149 & .811 & 2.914 & 49 & .005 \\
\hline
\end{tabular}

Table 2: Statistical Analysis of Cornell Medical Indices and Social Support Scores for Females.

\begin{tabular}{|c|c|c|c|c|c|c|c|c|c|}
\hline \multirow{3}{*}{$\begin{array}{l}\text { Pair } \\
\text { No. }\end{array}$} & \multirow{3}{*}{ System Studied } & \multicolumn{5}{|c|}{ Paired Differences } & \multirow{3}{*}{$\mathrm{t}$} & \multirow{3}{*}{$\mathrm{df}$} & \multirow{3}{*}{$\begin{array}{l}\text { Sig.(2- } \\
\text { tailed) }\end{array}$} \\
\hline & & \multirow[t]{2}{*}{ Mean } & \multirow[t]{2}{*}{$\begin{array}{c}\text { Std. } \\
\text { Deviation }\end{array}$} & \multirow{2}{*}{$\begin{array}{l}\text { Std. } \\
\text { Error } \\
\text { Mean }\end{array}$} & \multicolumn{2}{|c|}{$\begin{array}{l}95 \% \text { Confidence } \\
\text { Interval of the } \\
\text { Difference }\end{array}$} & & & \\
\hline & & & & & Lower & Upper & & & \\
\hline 1 & $\begin{array}{l}\text { Eyes and Ears (cases)- } \\
\text { Eyes and Ears (controls) }\end{array}$ & $\begin{array}{r}1.04 \\
.82\end{array}$ & $\begin{array}{l}1.245 \\
1.101\end{array}$ & $\begin{array}{l}.176 \\
.156\end{array}$ & -.233 & .673 & .976 & 49 & .334 \\
\hline 2 & $\begin{array}{l}\text { Respiratory (cases)- } \\
\text { Respiratory (controls) }\end{array}$ & $\begin{array}{r}1.28 \\
.68\end{array}$ & $\begin{array}{l}1.144 \\
1.133\end{array}$ & $\begin{array}{l}.162 \\
.160\end{array}$ & . 127. & 1.073 & 2.547 & 49 & .014 \\
\hline 3 & $\begin{array}{l}\text { Cardiovascular (cases)- } \\
\text { Cardiovascular (controls) }\end{array}$ & $\begin{array}{l}1.98 \\
1.12\end{array}$ & $\begin{array}{l}1.237 \\
1.304\end{array}$ & $\begin{array}{l}.175 \\
.184\end{array}$ & .401 & 1.319 & 3.762 & 49 & .000 \\
\hline 4 & $\begin{array}{l}\text { Gastrointestinal (cases)- } \\
\text { Gastrointestinal (controls) }\end{array}$ & $\begin{array}{l}1.90 \\
1.02\end{array}$ & $\begin{array}{l}1.359 \\
1.116\end{array}$ & $\begin{array}{l}.192 \\
.158\end{array}$ & .346 & 1.414 & 3.309 & 49 & .002 \\
\hline 5 & $\begin{array}{l}\text { Musculoskeletal (cases)- } \\
\text { Musculoskeletal (controls) }\end{array}$ & $\begin{array}{r}1.04 \\
.60\end{array}$ & $\begin{array}{r}1.261 \\
.833\end{array}$ & $\begin{array}{l}.178 \\
.118\end{array}$ & .025 & .855 & 2.132 & 49 & .038 \\
\hline 6 & $\begin{array}{l}\text { Skin (cases)- } \\
\text { Skin (controls) }\end{array}$ & $\begin{array}{l}.88 \\
.30\end{array}$ & $\begin{array}{r}1.206 \\
.707\end{array}$ & $\begin{array}{l}.171 \\
.100\end{array}$ & .154 & 1.006 & 2.735 & 49 & .009 \\
\hline 7 & $\begin{array}{l}\text { Nervous (cases)- } \\
\text { Nervous (controls) }\end{array}$ & $\begin{array}{l}1.56 \\
1.14\end{array}$ & $\begin{array}{l}1.264 \\
1.355\end{array}$ & $\begin{array}{l}.179 \\
.192\end{array}$ & -.119 & .959 & 1.566 & 49 & .124 \\
\hline
\end{tabular}


RELATIONSHIP OF PARENTAL HEALTH AND SOCIAL SUPPORT DURING PREGNANCY WITH THE RISK OF NON-SYNDROMIC ORAL

\begin{tabular}{|c|c|c|c|c|c|c|c|c|c|}
\hline 8 & $\begin{array}{l}\text { Genitourinary (cases)- } \\
\text { Genitourinary (controls) }\end{array}$ & $\begin{array}{r}1.66 \\
.88\end{array}$ & $\begin{array}{l}1.437 \\
1.172\end{array}$ & $\begin{array}{l}.203 \\
.166\end{array}$ & .253 & 1.307 & 2.974 & 49 & .005 \\
\hline 9 & $\begin{array}{l}\text { Fatigability (cases)- } \\
\text { Fatigability (controls) }\end{array}$ & $\begin{array}{r}1.30 \\
.56\end{array}$ & $\begin{array}{r}1.389 \\
.837\end{array}$ & $\begin{array}{l}.196 \\
.118\end{array}$ & .257 & 1.223 & 3.078 & 49 & .003 \\
\hline 10 & $\begin{array}{l}\text { Frequency of illness (cases)- } \\
\text { Frequency of illness } \\
\text { (controls) }\end{array}$ & $\begin{array}{l}.68 \\
.20\end{array}$ & $\begin{array}{r}1.203 \\
.535\end{array}$ & $\begin{array}{l}.170 \\
.076\end{array}$ & .130 & .830 & 2.753 & 49 & .008 \\
\hline 11 & $\begin{array}{l}\text { Miscellaneous diseases } \\
\text { (cases)-Miscellaneous } \\
\text { disease (controls) }\end{array}$ & $\begin{array}{r}1.28 \\
.92\end{array}$ & $\begin{array}{r}1.230 \\
.986\end{array}$ & $\begin{array}{l}.174 \\
.140\end{array}$ & -.065 & .785 & 1.703 & 49 & .095 \\
\hline 12 & $\begin{array}{l}\text { Health habits (cases)- } \\
\text { Health habits (controls) }\end{array}$ & $\begin{array}{l}.96 \\
.32\end{array}$ & $\begin{array}{r}1.124 \\
.653\end{array}$ & $\begin{array}{l}.159 \\
.092\end{array}$ & .252 & 1.028 & 3.311 & 49 & .002 \\
\hline 13 & $\begin{array}{l}\text { Inadequacy (cases)- } \\
\text { Inadequacy (controls) }\end{array}$ & $\begin{array}{r}1.48 \\
.88\end{array}$ & $\begin{array}{l}1.374 \\
1.304\end{array}$ & $\begin{array}{l}.194 \\
.184\end{array}$ & .061 & 1.139 & 2.239 & 49 & .030 \\
\hline 14 & $\begin{array}{l}\text { Depression (cases)- } \\
\text { Depression (controls) }\end{array}$ & $\begin{array}{r}1.20 \\
.48\end{array}$ & $\begin{array}{r}1.325 \\
.886\end{array}$ & $\begin{array}{l}.187 \\
.125\end{array}$ & .233 & 1.207 & 2.969 & 49 & .005 \\
\hline 15 & $\begin{array}{l}\text { Anxiety (cases)- } \\
\text { Anxiety (controls) }\end{array}$ & $\begin{array}{r}1.28 \\
.60\end{array}$ & $\begin{array}{l}1.371 \\
1.107\end{array}$ & $\begin{array}{l}.194 \\
.156\end{array}$ & .152 & 1.208 & 2.590 & 49 & .013 \\
\hline 16 & $\begin{array}{l}\text { Sensitivity (cases)-Sensitivity } \\
\text { (controls) }\end{array}$ & $\begin{array}{r}1.48 \\
.94\end{array}$ & $\begin{array}{l}1.418 \\
1.391\end{array}$ & $\begin{array}{l}.200 \\
.197\end{array}$ & -.071 & 1.151 & 1.776 & 49 & .082 \\
\hline 17 & $\begin{array}{l}\text { Anger (cases)- } \\
\text { Anger (controls) }\end{array}$ & $\begin{array}{r}1.40 \\
.66\end{array}$ & $\begin{array}{r}1.325 \\
.982\end{array}$ & $\begin{array}{l}.187 \\
.139\end{array}$ & .243 & 1.237 & 2.994 & 49 & .004 \\
\hline 18 & $\begin{array}{l}\text { Tension (cases)- } \\
\text { Tension (controls) }\end{array}$ & $\begin{array}{r}1.26 \\
.74\end{array}$ & $\begin{array}{l}1.226 \\
1.084\end{array}$ & $\begin{array}{l}.173 \\
.153\end{array}$ & .056 & .984 & 2.253 & 49 & .029 \\
\hline 19 & $\begin{array}{l}\text { Number of Persons available } \\
\text { (cases)-Number of persons } \\
\text { available (maximum:9) } \\
\text { (controls) }\end{array}$ & $\begin{array}{l}1.1082 \\
1.4552\end{array}$ & $\begin{array}{l}.50769 \\
.46933\end{array}$ & $\begin{array}{l}.07180 \\
.06637\end{array}$ & -.53325 & -.16075 & -3.744 & 49 & .000 \\
\hline 20 & $\begin{array}{l}\text { Level of Satisfaction (cases)- } \\
\text { Level of Satisfaction } \\
\text { (maximum:6) (controls) }\end{array}$ & $\begin{array}{l}4.9318 \\
5.4628\end{array}$ & $\begin{array}{l}.79474 \\
.40039\end{array}$ & $\begin{array}{l}.11239 \\
.05662\end{array}$ & -.77732 & -.28468 & -4.332 & 49 & .000 \\
\hline
\end{tabular}

\section{Discussion}

Oral clefts have been investigated by scholars from various fields. Our research on Pakistani population shows that status of health of both parents and moral support of family and society plays a leading role in determination of oral clefts.

Maternal and paternal ages are believed to be independent risk factors for oral clefts. Existing literature proposes an association between increasing paternal age and the risk but maternal age has not been highlighted as such. ${ }^{5-7}$ Our results also suggest an inclination along the same lines. However, we also find it mentionable that the data we collected has not been extensive enough to establish paternal age as a definite risk factor. Parental occupational exposures ${ }^{16}$ and low socioeconomic status are also considered as risk factors for oral clefts ${ }^{13}$. However, such effects were not observed in our study.

Although many researchers have pointed out other risk factors like alcohol consumption, smoking, and specific vitamin deficiencies, ${ }^{13,14}$ generalized parental health has rarely been discussed. In this study, it was seen that case parents had consistently poorer health status observed for each organ system subcategory. Studies conducted in past showed that smoking, ${ }^{13}$ domestic air pollutants (e.g. stoves), commercial inha- 
lants ${ }^{16}$ may be perceived as a provoking factor in causing respiratory problems and our study also showed promising results. Problems of musculoskeletal, cardiovascular, skin - related and genitourinary health and illness frequency also may be considered as indicators for the generalized health of parents and are probably coupled with excessive physical work, approach to health care parental health and availability of medicines. Maternal stress has also been discussed as a risk factor for such congenital anomalies. ${ }^{24}$ All of its possible causes can lead to raised blood glucocorticoid levels and high blood steroids may be linked to the risk of oral clefts. ${ }^{23}$ Maternal stress levels were explored by assessing maternal perception of her own mental health and behavior as well as by employing the Social Support questionnaire. The results were found to be significant enough to consider the social support during pregnancy as a contributor towards the said risk.

As predictable for most case control studies, especially in case of less commonly encountered health problems, the main limitations of this study have been smaller sample size and recall bias. The smaller sample size reduces the ability to comment surely upon the debatable risk factors like parental age. The children with oral clefts require early surgical intervention mostly and hence present to the hospital at an earlier age. The same may not be true for the controls. Moreover, the parental educational status, understanding and memory regarding their health status during the pregnancy under discussion had to be relied upon and there remains the possibility of discrepancies in the accuracy on these accounts among the cases and controls. However, each step was carefully carried out to allow minimum hampering of accurate data collection. Certain measures were taken like explaining the questionnaires to the patients in their own language and according to their level of understanding and asking questions in more than one ways, to ensure that the exercise would be reliable enough.

\section{Conclusion}

Oral clefts have a multi-factorial etiology. Hence, an effective preventive program should take into account parental health and social support factors.

\section{References}

1. [Online] Available from:
URL:http://www.medicinenet.com/script/main/art.asp? articlekey $=6538$

2. [Online] Available from:

URL:http://www.medicinenet.com/script/main/art.asp? articlekey $=6537$

3. Cleft Lip and Cleft Palate. [Online] Available from:URL:http://www.mayoclinic.org/diseasesconditions/cleft-palate/basics/complications/con20024619

4. Ganatra MA.Cleft Lip and Cleft Palate. Medicine Today, 2010; Vol. 8 (1): Pg no.14,15.

5. Herkrath APC Herkrath FJ Rebelo MAB Vettore MV. Parental Age as A Risk Factor For Non-Syndromic Clefts - A Meta Analysis. Journal of Dentistry, 2015; 54: 40 (1).

6. Fraser GR Calnan JS. Cleft Lip and Palate: Seasonal Incidence, Birth Weight, Birth Rank, Sex, Site, Associated Malformations and Parental Age. USA: National Center for Biotechnology Information, U.S. National Library of Medicine (internet). August 1961 (02 05 2015).

7. Woolf CM. Paternal Age Effect for Cleft Lip and Palate. USA: National Center for Biotechnology Information, U.S. National Library of Medicine (Internet); December 1963 (02 05 2015).

8. Mayo Clinic Staff. Risk factors. Diseases and Conditions Cleft Lip and Cleft Palate.

9. Jajja MRN Ghani A Cawasji F Sehyr I Khan MS Hashmi SS et al. Oral Clefts: A review of the cases and our experience at a single institution. Journal of Pakistan Medical Association (Internet), 201309 (2015 05 03).

10. Department of Health Services. Birth defect risk factor series: Oral Cleft. (Internet). 2005 Nov (2015 0503 ).

11. Qi L Liu J Zhang Y Wang J Yang M Gong T et al.Risk factors for non-syndromic oral clefts: a matched casecontrol study in Hubei Province, China. Wiley Online Library (internet). 20130919 (2015 05 03); Vol. 21 (1).

12. Gili JA Poletta FA Campaña H Comas B Pawluk M Rittler M et.al. Is Gravidity 4+ A Risk Factor For Oral Clefts? A Case Control Study In Eight South American Countries Using Structural Equation Modelling. The Cleft Palate - Craniofacial Journal [Online] 20139 (2015 5 4; 50 (5).

13. Jia Z Shi B Chen C Shi J Wu J Xu X. Maternal malnutrition, environmental exposure during pregnancy and the risk of non-syndromic orofacial clefts. Wiley Online Library (internet). 28 April 2011 (02 05 2015).

14. Lorente C Cordier S Goujardd J Aymé S Bianchi F Calzolari E, et al. Tobacco and alcohol use during pregnancy and risk of oral clefts. Occupational Exposure and Congenital Malformation Working Group. Am J Public Health, 2000 March; 90 (3): 415-419.

15. Källén B. Maternal Drug Use and Infant Cleft Lip / Palate with Special Reference to Corticoids. The Cleft 
Palate - Craniofacial Journal, 2003 Nov; 40 (6): pp. 624-628.

16. Lorente C, Cordier S, Bergret A, DE Welle Hek, Goujard J, Ayme S, et al. Maternal Occupational Risk Factors for Oral Clefts. Scand J. Work Environl Health, 2000; 26 (2): p137-145.

17. Ma J Huang YQ Yao C Ma SQ Meng T Ma M Su GH Zhai K Zhou ZW Zhu JF Shi B. Parental Health and Social Support in the First Trimester of Pregnancy and the Risk of Oral Clefts: A Questionnaire - Based, CaseControl Study. Plastic and Reconstructive Surgery, 2015 Jan; 135 (1): 212-8.

18. [Online]Available from:

URL:http://www.webmd.com/oral-health/guide/cleftlip-cleft-palate

19. Saal HM. Classification and Description of Non-Syndromic Cleft. In: Wyszynski DF. Cleft lip and Palate: From Origin to Treatment. New York: Oxford University Press; 2002: p47.

20. [Online] Available from: URL:

http://library.weill.cornell.edu/About/cornellmedindex. html
21. Sarason IG Levine HM Basham RB et al. Assessing social support: The Social Support Questionnaire. Journal of Personality and Social Psychology, 1983; 44: 127-139.

22. Vallino - Napoli LD Riley MM Halliday J. An Epidemiologic Study of Isolated Cleft Lip, Palate, or Both in Victoria, Australia From 1983 to 2000.The Cleft Palate - Craniofacial Journal, 2004 Mar; 41 (2): pp18594.

23. Carmichael SL, Shaw GM, Ma C, Werler MM, Rasmussen SA, Lammer EJ. Maternal corticosteroid use and orofacial clefts. American Journal of Obstetrics and Gynecology, 2007; 197 (6): pp581-5.

24. Carmichael SL Shaw GM Yang Abrams WB Lammer EJ. Maternal Stressful Life Events and Risks of Birth Defects. Carmichael SL, Shaw GM, Yang W, Abrams B, Lammer EJ. Maternal Stressful Life Events and Risks of Birth Defects. Epidemiology( Cambridge, Mass). 2007; 18 (3): pp356- 